\title{
Um resumo do estado da arte acerca da Síndrome Metabólica. Conceito, operacionalização, estratégias de análise estatística e sua associação a níveis distintos de actividade física
}

\author{
Joana Leal \\ Rui Garganta \\ André Seabra \\ Raquel Chaves \\ Michele Souza \\ José Maia
}

\author{
$\mathrm{CIFI}^{2} \mathrm{D}$ \\ Faculdade de Desporto \\ Universidade do Porto \\ Portugal
}

\section{RESUMO}

A Síndrome Metabólica (SM) resulta de um agrupamento de um conjunto de factores de risco cardiovascular. É uma enfermidade particularmente importante, que tem preocupado a comunidade médica e científica a nível mundial uma vez a sua prevalência está a aumentar gradualmente, tanto em adultos como em crianças, e está fortemente associada ao aumento do risco de desenvolvimento de doenças cardiovasculares e mortalidade.

Não existe ainda uma definição consensual sobre a SM, valores de corte para cada um dos indicadores e estratégias de análise estatística. Além disso, apesar de ser do conhecimento geral que a actividade física e o exercício físico têm um papel muito benéfico na prevenção e tratamento da SM, ainda não é clara a dose-resposta ideal.

É objectivo do presente estudo fazer uma revisão sumária sobre os critérios de definição da SM assim como esclarecer e clarificar as várias possibilidades de análise estatística e os seus impactos nos resultados obtidos utilizando diferentes abordagens. Conjuntamente pretende-se perceber qual a relação de diferentes níveis de (in)actividade física na manifestação da SM.

Palavras-chave: síndrome metabólica, critérios de definição, métodos de análise estatística, actividade física, aptidão cárdiorespiratória

\begin{abstract}
A brief summary of the state-of-the-art concerning the Metabolic Syndrome. Concept, measurement issues, data analysis strategies and associations with physical activity

The Metabolic syndrome (MS) is considered as a cluster of cardiovascular risk factors. It is a particularly important infirmity which concerns the medical and scientific community at a worldwide level as it has been increasing gradually in children and as well as in adults, and is strongly associated with the development of risk increases of cardiovascular diseases and mortality.

There is still no consensual definition about MS, cut-off values for every single indicator and unique statistical analysis strategies to deal with its results. Although it is generally accepted that physical activity and physical exercise have benign impacts on MS prevention and treatment, it is not yet well demonstrated the precise dose-response relationship. The main purpose of the state-of-the-art summary is to revise the MS definition, criteria to classify the MS and the varieties of statistical analysis. IN addition we shall focus on the relationship between physical activity and MS manifestations.
\end{abstract}

Key-words: metabolic syndrome, definition criteria, methods for statistic analysis, physical activity, cardio-respiratory fitness 


\section{INTRODUÇÃO}

Os tempos modernos estão intimamente associados a mudanças drásticas no estilo de vida, comportamentos e hábitos nutricionais das populações à escala mundial. Estas alterações, designadas de transições epidemiológicas, têm um forte impacto na saúde pública, nomeadamente no que diz respeito ao aumento do risco cardiovascular(63). É do conhecimento geral que as doenças cardiovasculares representam um dos mais graves problemas de saúde pública com consequências sérias para o doente, para a família e para a economia dos povos. Segundo a OMS (2007)(58), as doenças cardiovasculares posicionam-se como a causa número um de mortalidade global e estima-se que em 2015 cerca de 20 milhões de pessoas sofram mortalmente destas doenças. Em Portugal os números são igualmente preocupantes. Segundo a ACSS (Administração Central do Sistema de Saúde, em Portugal)(1), em 2006 o número de internamentos por enfarte agudo do miocárdio enfarte foi de 11943 dos quais $11,4 \%$ resultaram em óbito. Se se alargar a pesquisa para todas as patologias cardiovasculares (além do enfarte) verifica-se que de todos os óbitos registados em 2006 , cerca $36 \%$ foram de causa cardiovascular ${ }^{(28)}$. Se estes valores são preocupantes na perspectiva da saúde pública e da taxa de mortalidade, não se deve esquecer nunca o impacto económico que estas patologias implicam (para o doente, a família e o estado). Por exemplo, foi estimado que os recursos económicos gastos com as doenças cardiovasculares em 2003 em diferentes países europeus ascenderam a cerca de 169 biliões de euros em procedimentos de emergência, internamentos, recursos humanos, medicamentos e outros relacionados com estas doenças (33).

A tarefa de prevenir e combater as doenças cardiovasculares e diminuir os seus factores de risco, tem vindo a ser dificultada pelo facto de alguns desses factores se manifestarem de forma agrupada, isto é, em clusters. Isto acontece em alguns distúrbios de várias origens, especialmente nos metabólicos como é o caso da Síndrome Metabólica (SM) ${ }^{(45)}$. Esta síndrome tem impactos significativos a vários níveis, já que é notória a relação entre a SM e as doenças car-

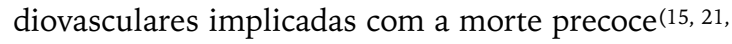
30). Além disso, a sua prevalência afecta diferentes estratos etários, sendo elevada tanto em adultos $(36$, 43), quanto em crianças e adolescentes (10, 20, 40, 49, 53). Estes números reforçam a necessidade de tomar medidas urgentes direccionadas à prevenção e tratamento destas doenças assim como a redução dos seus factores de risco. Face ao quadro traçado e dada a relevância emergente da $\mathrm{SM}$, o objectivo do presente estudo passa por apresentar a definição, actualização e clarificação de aspectos fundamentais da síndrome metabólica de que destacamos:

- Revelar o trajecto histórico da definição desta síndrome, desde a sua primeira sinalização até aos dias de hoje.

- dentificar os critérios de classificação mais actuais da SM e os problemas decorrentes da diversidade de critérios.

- Apresentar os principais procedimentos de análise estatística da SM.

- Descrever a associação de níveis distintos da actividade física com o valor contínuo de SM

\section{A NOÇÃO DE SÍNDROME (METABÓLICA)}

A síndrome metabólica, o seu conceito, operacionalização, relevância enquanto entidade clínica e impacto na saúde das populações, são assuntos de forte debate ${ }^{(62)}$. Contudo, antes de abordarmos os aspectos fundamentais da sua definição operacional e formas de análise estatística dos resultados de diferentes pesquisas, é importante relembrar o sentido etimológico da expressão "síndrome metabólica". O significado do termo síndrome é ele próprio fonte de controvérsia, sugerindo diversos questionamentos sobre seu significado clínico.

Síndrome é uma palavra de origem grega que assume várias formas geradas de pequenas alterações da própria palavra inicial - syndromé - que queria designar conjunto(47). De acordo com este autor, o termo terá sido usado pela primeira vez na Grécia no século XVI, e terá assumido o significado de um conjunto de sintomas sem relação obrigatória com determinada doença.

Em inglês, a palavra "syndrome" terá entrado para o vocabulário médico em meados de 1541, após uma tradução das obras de Galeno. A grafia terá sido mantida com acentuação na antepenúltima sílaba tal como na língua castelhana e italiana. Já na língua Portuguesa, surgiram cinco variações desta mesma 
palavra, "síndrome", "sindroma", "síndromo", "sindromo" e "síndroma", todas elas com o mesmo significado. Actualmente, ficou estabelecido que a forma "síndrome" (pela aproximação à origem grega syndromé) ou mesmo "síndroma”, acentuadas na antepenúltima sílaba, e de género feminino, são as formas correctas e aceites em linguagem médica Portuguesa(22).

No que concerne ao seu significado clínico, a SM é um cluster de factores de risco de variáveis somáticas, fisiológicas e bioquímicas, associados à processos mórbidos, como diabetes tipo II e doenças cardiovasculares, que juntos formam o quadro clínico dessa síndrome ${ }^{(21)}$.

\section{ASPECTOS HISTÓRICOS}

Foi nos anos 20 que se começou a atribuir maior importância à associação de alguns factores de risco cardiovascular com a resistência à insulina. No entanto, teríamos que esperar até 1988 para entender com maior clareza a entidade hoje conhecida por síndrome metabólica (SM). Foi Gerald Reaven(44) que nas Banting Lectures reclamou atenção para o facto de alguns factores de risco cardiovasculares, nomeadamente resistência à insulina, obesidade abdominal, baixos valores de lipoproteínas de alta intensidade (C-HDL), triglicerídeos e hipertensão arterial, aparecerem com frequência de forma agrupada em alguns indivíduos. Esta condição foi denominada por síndrome de resistência à insulina, pois todos os indivíduos que possuíam este estranho "cluster" de diferentes factores de risco, tinham baixa sensibilidade à insulina. Esta síndrome tem sido fértil em derivações: Síndrome X, síndrome X de Reaven, Síndrome plurimetabólica, quarteto mortal, síndrome X metabólica, síndrome X plus, hipertrigliceridemia abdominal $(17,31,42)$.

É consensual que a síndrome está associada ao aumento de risco cardiovascular a longo prazo, diabetes tipo II, patologia hepática (não alcoólica), que varia de esteatose macrovesicular simples, esteatoepatite, fibrose avançada e cirrose, patologia renal, glomerulopatia, e alguns tipos de $\operatorname{cancro}(15,22,28)$. Esses factores de risco são maioritariamente de origem metabólica. No entanto, existem outros factores associados que potenciam o aumento e a severidade dos indicadores metabólicos de que destacamos a inactividade física, a obesidade, a idade, e ainda os hábitos nutricionais inadequados (23).

A etiologia da SM não é ainda totalmente conhecida, bem como todos os mecanismos de sua fisiopatologia(45). Contudo, é consensual que resulta da interacção entre factores metabólicos, genéticos e ambientais $(6,7$, $12,17,24,34,37,38,39,46,51,52)$. Exactamente por este motivo, não existe ainda uma definição universal a que se associem indicadores e valores de corte únicos.

Muitos autores avançaram com definições que foram sendo completadas/corrigidas ao longo dos anos. Várias organizações académicas e profissionais, e grupos de estudo apresentaram as suas posições oficiais acerca da SM de que destacamos a OMS (1998)(2), a EGIR (1999)(8), a NCEP-ATP III $(2001)^{(19)}$, a AACE/ACE (2003) (4) e mais recentemente a IDF (2005) (3).

A OMS (1998) foi a primeira organização a avançar com uma definição oficial da síndrome metabólica, e a reunir um conjunto de critérios de diagnóstico muito específicos. O pressuposto principal centra-se no facto da resistência à insulina ou a presença de um qualquer distúrbio do metabolismo da glicose ser um dos maiores contribuintes para a SM. Assim, a resistência à insulina foi considerada pela OMS (1998) como o factor principal para o diagnóstico. Além disso, seriam necessários a coexistência de, pelo menos, mais dois factores de risco (ver Tabela 1) para que o diagnóstico fosse feito. Esses factores de risco têm valores de corte muito específicos, que em 1999 foram sujeitos a alguns ajustes nomeadamente no que diz respeito aos valores da pressão arterial(56). Todos os restantes aspectos foram mantidos. Incluíram ainda um outro parâmetro como componente de diagnóstico - a micro albuminúria - que gerou imediatamente controvérsia por parte de várias outras organizações.

Em 1999 a EGIR - The European Group for the Study of Insulin Resistence ${ }^{(8)}$ propôs uma versão modificada da anterior que poderia ser usada em indivíduos não diabéticos. Defendiam a denominação de Síndrome de Resistência à Insulina em vez de SM pois, tal como a OMS, também assumiam que a resistência à insulina fosse a causa mais relevante para o diagnóstico. Tal como a OMS, além da resistência à insulina, deveria ser integrado no diagnóstico a presença de mais dois factores de risco. De todos os indicadores 
na Tabela 1, deram particular atenção à obesidade abdominal. Para estimar a resistência à insulina, sugeriram o uso dos níveis de insulina plasmática e/ou glucose em jejum. Além disso, eram considerados outros factores como o facto dos sujeitos estarem a ser tratados a dislipidemias ou a hipertensão, por evidências da associação de tais desordens com as doenças cardiovasculares.

Uma terceira entidade, a National Cholesterol Education Program's Adult Treatment Panel III (NCEP-ATPIII, 2001) (19) propôs uma nova definição da SM e reformulando os critérios de avaliação dos diferentes indicadores, com o objectivo de facilitar o diagnóstico e a prática clínica. Diferia das anteriores em dois grandes pontos: em primeiro lugar não via a resistência à insulina como factor principal e exclusivo, com a possibilidade de não ser representativa do estilo de vida do indivíduo); em segundo lugar não se atribuía uma grande ênfase aos valores da glicose, tratando-os de igual forma em relação a todos os outros componentes. Referiu a agregação de pelo menos três dos cinco factores de risco, com valores de corte muito específicos (Tabela 1). Além disso, era tida em conta a análise cuidada e acompanhamento clínico de indivíduos com apenas dois factores de risco, uma vez que alguns indivíduos pareciam ser igualmente insulino-resistentes tais como os indivíduos com três ou mais factores. Este aspecto permitia estender o diagnóstico de SM a outros sujeitos que não tivessem exclusivamente os três ou mais factores de risco, mas a todos os que tivessem apenas dois factores e uma história clínica compatível com a SM.

Em 2003, a American Association of Clinical Endocrinology (AACE) juntamente com a American College of Endocrinology (ACE) (4) manifestaram-se igualmente sobre este assunto. As atenções foram redireccionadas para a resistência à insulina como principal factor de risco cardiovascular e redefiniu-a como Síndrome de Resistência à insulina (tal como a EGIR). Integraram no seu documento, vários outros factores que iriam identificar este síndrome mas não definiu nenhum número mínimo para que o diagnóstico fosse realizado. A grande diferença reside na ênfase dada ao diagnóstico baseado na história clínica, história familiar e anamnese, não havendo uma nova definição para a SM, mas sim maior importância à resistência à insulina.
A última entidade a pronunciar-se acerca da definição e critérios de caracterização da síndrome metabólica foi a International Diabetes Foundation (IDF) que publicou a sua posição em Dezembro de 2005(3). As grandes diferenças para as entidades anteriores residiram no facto de considerarem o perímetro abdominal (obesidade abdominal) como critério fundamental, cujos valores situam-se entre $\geq 85$ a $94 \mathrm{~cm}$ para homens e $\geq 80$ a $90 \mathrm{~cm}$ para mulheres, sendo específicos a diversas etnias, para o diagnóstico da síndrome metabólica, uma vez que esta medida era considerada altamente correlacionada com a resistência à insulina. Assim sendo, as medidas laboratoriais da resistência à insulina tornam-se desnecessárias. Além do perímetro abdominal, é condição imprescindível para a realização do diagnóstico, a presença de mais dois factores de risco. A definição da IDF chama ainda à atenção para o facto dos valores do perímetro abdominal necessitarem de ser ajustados a indivíduos de nacionalidades e etnias diferentes. Assim, para esses casos, é aconselhado o uso de dados de estudos já realizados em populações específicas.

Além dos factores anteriores, muitos outros foram relacionados com a síndrome metabólica. Pela escassez de estudos sobre os mesmos, a maioria não é incluída nos critérios de definição da síndrome. Grundy (23) e Terán-Garcia(51) salientaram:

- Distribuição anormal de massa gorda (geral, central, biomarcadores de tecido adiposo, índice de gordura hepática, gordura miocelular)

- Dislipidemia, além dos valores elevados de colesterol total, lipoproteínas de baixa densidade (LDL) e triglicerídeos (Apoliproteina B, LDL, rácio Triglicerídeos/HDL)

- Resistência à insulina, além dos valores da glicose em jejum (Insulina em jejum, HOMA - IR: Homeostasis Model Assessment for Insuline Resistance, Bergman Minimal Model, valores elevados de ácidos gordos livres.)

- Desregulação vascular

- Estado Pró-Infamatório (Proteína reactiva C de elevada sensibilidade, concentrações elevadas e valores baixos de citocinas

- Estado pró-trombótico (factores coagulantes, factores fibrinolíticos)

- Outros factores hormonais (eixo corticoesteróide, síndrome do ovário policístico). 
Tabela 1. Indicadores e critérios para a definição da SM [adultos].

\begin{tabular}{|c|c|c|c|c|c|}
\hline & OMS (1999) & EGIR (1999) & NCEP ATPIII (2001) & AACE (2002) & IDF (2005) \\
\hline Critérios de & IGT, IFG, DM 2 ou & Insulina Plasmática & Sem relevância & IGT ou IFG & Sem relevância \\
\hline diagnóstico & baixa sensibilidade & $>P>5$ & 3 Factores seguintes & + & \\
\hline Resistência & à insulina & + & & Qualquer um dos 5 & \\
\hline \multirow[t]{3}{*}{ à Insulina } & + & 2 Factores seguintes & & factores analisados & \\
\hline & 2 Factores seguintes & & & com base em & \\
\hline & & & & avaliação clínica & \\
\hline Índices & Rácio cintura/anca & Perímetro da cintura: & Perímetro da cintura: & $I M C \geq 25 \mathrm{Kg} / \mathrm{m}^{2}$ & Aumento do períme- \\
\hline \multirow[t]{4}{*}{ Antropométricos } & $\sigma^{\nearrow}>90$ & $\sigma^{\top} \geq 94 \mathrm{~cm}$ & $\sigma^{\nearrow} \geq 102 \mathrm{~cm}$ & & tro abdominal (popu- \\
\hline & 우 > 85 & 우 $\geq 80 \mathrm{~cm}$ & 우 $\geq 82 \mathrm{~cm}$ & & lações específicas] \\
\hline & e/ou & & & & + \\
\hline & $\mathrm{IMC}>30 \mathrm{Kg} / \mathrm{m}^{2}$ & & & & 2 Factores seguintes \\
\hline \multirow[t]{8}{*}{ Lípidos } & Triglicerídeos: & Triglicerídeos: & Triglicerídeos: & Triglicerídeos: & Triglicerídeos: \\
\hline & $\geq 150 \mathrm{mg} / \mathrm{dL}$ & $\geq 150 \mathrm{mg} / \mathrm{dL}$ & $\geq 150 \mathrm{mg} / \mathrm{dL}$ & $\geq 150 \mathrm{mg} / \mathrm{dL}$ & $\geq 150 \mathrm{mg} / \mathrm{dL}$ \\
\hline & e/ou & e/ou & e/ou & e/ou & ou triglicerídeos RX \\
\hline & HDL: & HDL: & HDL: & HDL: & e/ou \\
\hline & $\sigma^{\top}<35 \mathrm{mg} / \mathrm{dl}$ & $<39 \mathrm{mg} / \mathrm{dl} \sigma^{\pi}$ ou 우 & $\sigma^{\top}<40 \mathrm{mg} / \mathrm{dl}$ & $\sigma^{\top}<40 \mathrm{mg} / \mathrm{dl}$ & HDL: \\
\hline & 우 < 39mg/dL & & 우 $<50 \mathrm{mg} / \mathrm{dL}$ & 우 < 50mg/dL & $\sigma^{\top}<40 \mathrm{mg} / \mathrm{dl}$ \\
\hline & & & & & 우 < 50mg/dL \\
\hline & & & & & Ou HDL RX \\
\hline \multirow[t]{3}{*}{ Pressão Arterial } & $\geq 140 / 90 \mathrm{mmHg}$ & $\geq 140 / 90 \mathrm{mmHg}$ & $\geq 130 / 85 \mathrm{mmHg}$ & $\geq 130 / 85 \mathrm{mmHg}$ & $\geq 130 / 85 \mathrm{mmHg}$ \\
\hline & & Ou a realizar & & & ou Hipertensão RX \\
\hline & & tratamento & & & \\
\hline \multirow[t]{2}{*}{ Glucose } & IGT, IFG ou DM2 & IGT ou IFG & $>110 \mathrm{mg} / \mathrm{dL}$ & IGT ou IFG & $\geq 100 \mathrm{mg} / \mathrm{dL}$ \\
\hline & & (não inclui diabetes] & (inclui diabetes) & (não inclui diabetes) & (inclui diabetes) \\
\hline \multirow[t]{3}{*}{ Outros } & Microalbuminúria & & & & \\
\hline & Excreção Urinária & & & & \\
\hline & $\mathrm{de}>20 \mu \mathrm{g} / \mathrm{min}$ & & & & \\
\hline
\end{tabular}

Abreviaturas: IGT - Impaired Glucose Tolerance (tolerância à glucose diminuída); IFG - Insuline Factor Growth (factor de crescimento semelhante a insulina); DM 2 diabetes mellitus 2; IMC - Índice de Massa Corporal; HDL - Colesterol com Lipoproteina de Alta Densidade; RX - a realizar tratamento específico para; P - Percentil

\section{DEFINIÇÃO E VALORES DE CORTE}

A Tabela 1 resume os indicadores e critérios para a definição da SM segundo várias organizações mundiais.

Como se verifica, as cinco organizações descrevem minuciosamente os critérios de classificação assim como os valores de corte. No entanto, apenas duas delas se manifestaram relativamente à SM em crianças e jovens: OMS e NCEP ATPIII, cujas propostas estão referidas na Tabela 2.
Outros autores também se pronunciaram acerca da $\mathrm{SM}$ em crianças e jovens. A Tabela 3 resume as referências mais actuais.

\section{DISCREPÂNCIA E VARIABILIDADE DOS CRITÉRIOS DE CLASSIFICAÇÃO}

A variabilidade associada aos valores de corte nos indicadores da SM conduz a resultados não consensuais quando se utilizam critérios distintos. Basta existirem indivíduos que, em função de uma classifi- 
Tabela 2. Indicadores e critérios para a definição da SM (crianças e jovens]. Comparação entre a OMS, NCEP ATPIII e IDF.

\begin{tabular}{|c|c|c|c|c|}
\hline & OMS (1999) & NCEP ATPIII (2001) & \multicolumn{2}{|c|}{ IDF (2007) (Zimmet, Alberti et al) (57) } \\
\hline $\begin{array}{l}\text { Critérios de } \\
\text { diagnóstico }\end{array}$ & $\begin{array}{l}\text { Agregação de } 3 \text { Factores } \\
\text { dos } 5 \text { seguintes }\end{array}$ & $\begin{array}{c}\text { Agregação de } 3 \text { Factores } \\
\text { dos } 5 \text { seguintes }\end{array}$ & 6-10 anos & $10-16$ anos \\
\hline Resistência & $\mathrm{Ou}$ & & & \\
\hline à Insulina & $\begin{array}{l}\text { Hiperinsulinemia } \\
+2 \text { factores }\end{array}$ & & & \\
\hline Peso corporal & $\begin{array}{c}\mathrm{IMC} \geq \mathrm{P} 75 / 85 / 95 \\
\text { consoante idade e sexo }\end{array}$ & $\begin{array}{c}\mathrm{PC}>\mathrm{P} 90 \\
\mathrm{Ou} \mathrm{IMC} \geq \mathrm{Pg}\end{array}$ & $\mathrm{PC}>\mathrm{PgO}$ & $\begin{array}{c}\mathrm{PC}>\mathrm{P} 90 \text { (ou referência } \\
\text { adulta se inferior) }\end{array}$ \\
\hline Lípidos & $\begin{array}{c}\text { Triglicerídeos: } \\
\geq 104 / 130 / 150 \mathrm{mg} / \mathrm{dL} \\
\text { ou } \geq \mathrm{P} 75 / 80 / 90 \\
\text { consoante idade e sexo } \\
\text { HDL: } \\
<35-40 \mathrm{mg} / \mathrm{dL} \text { ou }<\mathrm{P} 25 \\
\text { consoante idade e sexo }\end{array}$ & $\begin{array}{c}\text { Triglicerídeos: } \\
\geq 97 / 110 / 150 \mathrm{mg} / \mathrm{dL} \\
\text { ou } \geq \mathrm{P} 75 / 80 / 90 \\
\text { consoante idade e sexo } \\
\text { HDL: } \\
<35-40 \mathrm{mg} / \mathrm{dL} \text { ou }<\mathrm{P} 25 \\
\text { consoante idade e sexo }\end{array}$ & $\begin{array}{c}\text { Todos os restantes } \\
\text { indicadores não são } \\
\text { medidos. } \\
\text { A SM não deve ser } \\
\text { diagnosticada antes dos } \\
10 \text { anos. } \\
\text { A obtenção de dados }\end{array}$ & $\begin{array}{c}\text { Triglicerídeos: } \\
\geq 1.7 \mathrm{mmol} / \mathrm{L} \\
\mathrm{HDL}: \\
<1.03 \mathrm{mmol} / \mathrm{L}\end{array}$ \\
\hline Pressão Sanguínea & $\begin{array}{l}\geq \text { P75/80/95 consoante } \\
\text { idade, sexo e peso }\end{array}$ & $\begin{array}{l}\geq P 90 / 95 \text { consoante } \\
\text { idade, sexo e peso }\end{array}$ & $\begin{array}{l}\text { importantes para registo e } \\
\text { prevenção é dada pela }\end{array}$ & $\begin{array}{l}\text { Sistólica } \geq 130 \mathrm{mmHg} \\
\text { Diastólica } \geq 85 \mathrm{mmHg}\end{array}$ \\
\hline Glucose & $\begin{array}{c}\geq 110 \text { ou } \geq \mathrm{P} 80 / 90 \\
\text { consoante a idade, sexo } \\
\text { e diabetes }\end{array}$ & $\begin{array}{c}100 \text { ou } 110 \text { (em Jejum) } \\
\text { ou } \\
\geq 140 \text { 2h após ingestão }\end{array}$ & $\begin{array}{c}\text { Distorla famillar de SM, } \\
\text { cardiovascular } \\
\text { dislipidemias, hipertensão }\end{array}$ & $\begin{array}{c}\geq 5.6 \mathrm{mmol} / \mathrm{L} \\
\text { OU } \\
\text { DB2 diagnosticado }\end{array}$ \\
\hline Outros & $\begin{array}{c}\text { Insulina em Jejum } \\
\geq \text { P75/80/95 consoante } \\
\text { sexo, idade e raça }\end{array}$ & & ou obesidade. & \\
\hline
\end{tabular}

cação ou outra possam ser classificados como portadores ou não desta síndrome. Por exemplo, Dekker et al(15) realizou um estudo numa mesma população em que aplicou os critérios de definição operacional da NCEP, ATPIII, OMS, EGIR e ACE. Os resultados revelaram que, segundo os critérios da NCAP ATPIII, os indivíduos apresentavam um risco ligeiramente mais elevado quando comparado com os restantes. Isto poderá dever-se ao facto dos valores de corte impostos pela NCEP ATPIII implicarem um intervalo mais alargado de possibilidades, podendo eventualmente incluir um maior número de indivíduos.

Wijndaele(55) reforça esta dificuldade e demonstra as diferenças encontradas após um estudo de prevalência numa população Australiana usando os critérios da OMS, ATP III e EGIR. Os resultados foram igualmente desconcertantes.

Verifica-se uma grande discrepância entre os valores das prevalências, sendo que a maior diferença chega aos 4,9\% (OMS e EGIR). Adicionam-se ainda outros aspectos que dificultam a comparação de dados de diferentes estudos, que geram confusão em termos epidemiológicos. Pesquisas com diferentes delineamentos, populações com características físicas muito específicas, o ano de recolha dos dados e os critérios usados, a distribuição da amostra nomeadamente no que diz respeito a idade, "raça" e sexo geram problemas acrescidos na comparação de resultados.

Verifica-se, por exemplo, que em estudos de prevalências encontram-se resultados distintos, mas ao 
Tabela 3. Critérios de Classificação da síndrome metabólica em crianças e jovens. Diferenças entre os vários autores.

\begin{tabular}{|c|c|c|c|c|}
\hline & Cook et al (13) & Cruz et al (14] & Weiss et al (53) & Ferranti et al (20) \\
\hline Glicose & Em Jejum = 110 mg/dL & $\begin{array}{l}\text { 2h após ingestão } \\
\quad>140 \mathrm{mg} / \mathrm{dL}\end{array}$ & $\begin{array}{l}\text { 2h após ingestão } \\
\quad>140 \mathrm{mg} / \mathrm{dL}\end{array}$ & Em Jejum $=110 \mathrm{mg} / \mathrm{dL}$ \\
\hline Obesidade & $\begin{array}{l}\mathrm{PC}>\mathrm{P} 90 \text { consoante } \\
\text { idade e sexo }\end{array}$ & $\begin{array}{c}\mathrm{PC}>\mathrm{P} 90 \text { consoante } \\
\text { idade e sexo }\end{array}$ & $\begin{array}{c}\text { IMC > P97 consoante } \\
\text { idade e sexo }\end{array}$ & $\begin{array}{c}\mathrm{PC}>\mathrm{P} 75 \text { consoante } \\
\text { idade e sexo }\end{array}$ \\
\hline Triglicerídeos & $=110 \mathrm{mg} / \mathrm{dL}$ & $\begin{array}{l}\text { > P90 consoante } \\
\text { idade e sexo }\end{array}$ & $\begin{array}{l}\text { P95 consoante } \\
\text { idade e sexo }\end{array}$ & $=100 \mathrm{mg} / \mathrm{dl}$ \\
\hline C-HDL & $<40 \mathrm{mg} / \mathrm{dL}$ & $\begin{array}{c}<\text { P10 consoante } \\
\text { idade e sexo }\end{array}$ & $\begin{array}{c}<\text { P10 consoante } \\
\text { idade e sexo }\end{array}$ & $\begin{array}{l}<50 \mathrm{mg} / \mathrm{dl} \text { (excepto } \sigma^{\top} \\
15-19 \text { anos: }<45 \mathrm{mg} / \mathrm{dl})\end{array}$ \\
\hline Pressão arterial & $\begin{array}{c}\text { Sist. e Diast. > P90 } \\
\text { Consoante idade e sexo }\end{array}$ & $\begin{array}{c}\text { Sist. e Diast > P90 } \\
\text { Consoante idade e sexo }\end{array}$ & $\begin{array}{c}\text { Sist. e Diast > P95 } \\
\text { Consoante idade e sexo }\end{array}$ & $\begin{array}{c}\text { Sist. e Diast > P90 } \\
\text { Consoante idade e sexo }\end{array}$ \\
\hline
\end{tabular}

Abreviaturas: PC - Perímetro da cintura; IMC - Índice de Massa Corporal; C - HDL - Colesterol com Lipoproteina

de Alta Densidade; P - Percentil; Sist. - Pressão arterial Sistólica; Diast - Pressão arterial Diastólica

\section{Prevalência da Síndrome Metabólica numa População Australiana Não Diabética (2005)}

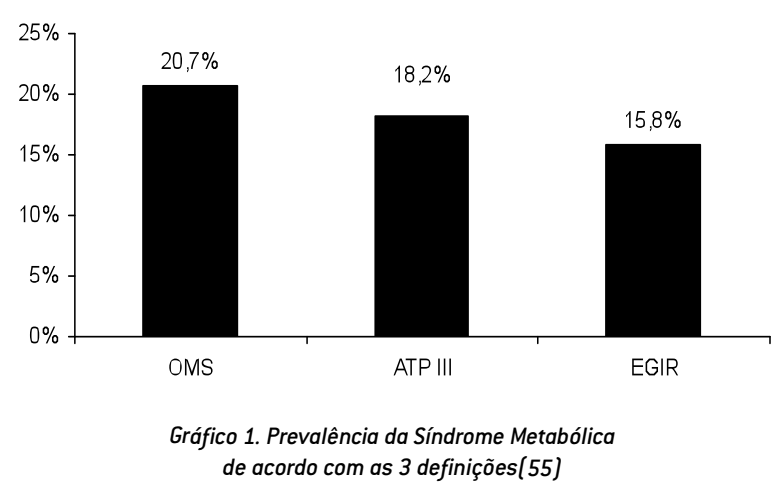

mesmo tempo interessantes quando analisamos diferentes populações. Por exemplo, em pesquisas cuja amostra é constituída por indivíduos entre os 20 e os 25 anos de idade e mais velhos, as prevalências variam desde os $8 \%$ (Índia) até aos $24 \%$ (EUA) nos homens, e desde 7\% (França) aos 43\% (Irão) nas mulheres. Na mesma população os resultados são diferentes se se distinguir grupos de raça/etnia diferente. Além disso, encontram-se variações a nível do sexo pois se numa população com ajuste à idade a prevalência de SM é mais elevada nas mulheres, noutras populações já é mais elevada nos homens(31).
A falta de homogeneidade na definição, nos critérios, características da amostra e tipo de estudo realizado, gera alguma confusão, tornando a investigação e comparação mais problemática. Alguns autores, tal como Yang(59) tentam contornar esta dificuldade usando no mesmo estudo, as três diferentes classificações e apresentam os resultados em função de cada uma delas.

Além deste problema, que implica um desacordo entre ter ou não ter síndrome metabólica, maior ou menor risco cardiovascular, existe um factor comum a todas as variações dos critérios de definição. Todos possuem valores de corte muito específicos, reduzindo a distribuição dos valores de todas as variáveis contínuas a uma dicotomização básica com perda de poder interpretativo cujas implicações clínicas e significado estatístico em termos epidemiológicos podem não ser as mais adequadas ${ }^{(54)}$.

Esta caracterização em "ter ou não ter" é muito redutora e limitativa uma vez que, se estamos a falar de uma doença progressiva que associa vários factores de risco agrupados e que se manifestam de forma diferente em função da presença uns dos outros, torna obviamente esta classificação muito pobre e sem aplicabilidade clínica. Não se percebe o verdadeiro risco que determinado indivíduo apresenta, nem a sua evolução/regressão face a um tratamento, 
por exemplo, nem se consegue distinguir grupos de indivíduos face a determinadas características físicas porque todos eles apenas "têm ou não têm" Síndrome Metabólica.

\section{MÉTODOS DE ANÁLISE ESTATÍSTICA E TRATAMENTO DOS DADOS DA SM}

A análise estatística dos diferentes indicadores da SM tem conduzido os investigadores a visões distintas da mesma informação. Neste sentido parece haver quatro estratégias de análise de dados:

1. Centrada nos valores contínuos dos indicadores da SM, com importância na fase inicial dos estudos onde se realiza uma forte análise exploratória e descritiva dos valores, com o propósito de providenciar informação epidemiológica básica relevante.

2. Centrada nos pontos de corte propostos reduzindo os valores contínuos das variáveis a entidades discretas, isto é a valores binários de grande utilidade em pesquisa epidemiológica, sobretudo no cálculo de prevalências, de grande relevância em termos clínicos.

3. Centrada na transformação de valores contínuos em scores $Z$, que depois de somados, permitem obter um score contínuo que tenta expressar a magnitude do valor da SM. Com base neste resultado é possível associá-lo a quartis e quintis de actividade física e capacidade cardio-respiratória.

4. Centrada na análise factorial (AF) e análise de componentes principais (ACP) com o objectivo de identificar as componentes ou factores principais que melhor descrevem as relações, isto é, a covariação entre os indicadores da SM.

A Tabela 4 apresenta um sumário brevíssimo de alguns estudos que recorrem às três principais formas de análise estatística da SM.

A primeira opção (de dicotomização de valores contínuos) tal como foi referido anteriormente, apresenta alguma insuficiência no que concerne ao poder estatístico dos resultados, bem como ao seu significado clínico(35). A este último respeito, o risco de desenvolver uma patologia cardiovascular ou apresentar síndrome metabólica apresenta-se sempre de modo progressivo(31). Ora esta progressão não impli$\mathrm{ca}$, necessariamente, um aumento do número de factores de risco, mas sim alteração nos resultados, isto é, aumento dos valores relativamente às referências, dos factores de risco(32). Daqui que, a análise da síndrome metabólica considerada como uma simples questão de "ter ou não ter" pode revelar-se redutora e simplista.

A segunda opção de análise surgiu na tentativa de ultrapassar o problema do reduzido poder estatístico da dicotomização das variáveis. A ideia base é construir um score (contínuo) de síndrome metabólica, calculado através da soma dos scores $z$ dos diferentes indicadores. Este score contínuo, é estatisticamente mais sensível na sua globalidade para expressar a SM do que as variáveis dicotómicas ${ }^{(54)}$. Este procedimento foi utilizado por diversos autores, como uma forma de ultrapassar a "simplicidade" do recurso a variáveis binárias $(5,9,21,48)$. Contudo, a simples soma dos valores $z$ não considerava a circunstância dos indicadores poderem ser pesados de modo distinto. Uma forma de resolver este problema foi sugerida por Wijndaele e colaboradores ${ }^{(54)}$, recorrendo à inclusão do valor da variância explicada pelas componentes principais que "aglutinam" os diferentes indicadores da síndrome.

Uma terceira forma lidar com os indicadores da síndrome metabólica recorre à análise factorial (AF) ou análise em componentes principais (ACP). O objectivo deste procedimento é identificar as associações e independência das diversas variáveis assim como verificar quais delas melhor explicam a covariância existente. Esta abordagem permite retirar informação complementar face às anteriores, uma vez que as características inerentes aos procedimentos estatísticos específicos que estão implicados são igualmente diferentes.

As diferentes formas de avaliar e interpretar os dados da SM são utilizadas em função dos objectivos de cada estudo. No entanto, esta variedade de procedimentos de análises acarreta uma multiplicidade de respostas que, se por um lado podem resultar numa agregação e compilação de conclusões interessantes, por outro poderá gerar controvérsia ou não responder à questão central de cada pesquisa. Além disso, e após analisar diversos estudos, começam a surgir novas questões, que pela sua complexidade não são bem esclarecidas com os modelos de análise anteriormente descritos. Atentemos no seguinte: os critérios de definição da síndrome metabólica têm, em geral, quatro ou cinco componentes. A maioria dos 
Tabela 4. Sumário de alguns estudos relativos às diferentes estratégias de análise da SM

\begin{tabular}{|c|c|c|c|c|c|}
\hline$\overline{\text { Autor (data) }}$ & Objectivos do estudo & País & Definição de SM & Amostra & Principais resultados \\
\hline & \multicolumn{5}{|c|}{ Dicotomização das variáveis - Valores de corte propostos } \\
\hline $\begin{array}{l}\text { Cook } \\
\text { et al (13) }\end{array}$ & $\begin{array}{c}\text { Prev. de SM } \\
\text { em adolescentes } \\
\text { nos EUA }\end{array}$ & EUA & $\begin{array}{l}\text { NCEP ATP III } \\
\text { (adaptado } \\
\text { para idade) }\end{array}$ & $\begin{array}{c}\mathrm{n}=2430 ; \text { Idade: } 12-19 \\
\sigma^{\top} 1150 \text { e 우 } 1280 \\
\text { Caucasianos (646), } \\
\text { Negros (824) e } \\
\text { mexico-americanos (846) }\end{array}$ & $\begin{array}{l}\text { Prev. total na } \\
\text { amostra: } 4,2 \%\end{array}$ \\
\hline $\begin{array}{l}\text { Yoshinaga } \\
\text { et al }(60)\end{array}$ & $\begin{array}{c}\text { Prev. de SM e diferenças } \\
\text { entre os sexos em crianças } \\
\text { obesas ou com Sp no Japão }\end{array}$ & Japão & $\begin{array}{l}\text { NCEP ATP III } \\
\text { (adaptado) }\end{array}$ & $\begin{array}{c}\mathrm{n}=1052 ; \text { Idade: } 6-12 \\
\sigma^{\top} \quad 674 \text { e 우 } 378 \\
172 \text { Sp e } 299 \text { obesas }\end{array}$ & $\begin{array}{l}\text { Prev. em obesas: } 17.7 \% \\
\quad \text { Prev. em Sp: } 8,7 \% \\
\text { Existem diferenças entre } \\
\text { sexos no que diz respeito } \\
\text { ao PC e na insulina }\end{array}$ \\
\hline $\begin{array}{l}\text { Jebb } \\
\text { et al (29) }\end{array}$ & $\begin{array}{c}\text { Prev. de SM e diferenças } \\
\text { entre os sexos em crianças } \\
\text { obesas ou com Sp na Grã- } \\
\text { Bretanha }\end{array}$ & Grã - Bretanha & $\begin{array}{l}\text { NCEP ATP III } \\
\text { (adaptado) }\end{array}$ & $\begin{array}{c}\mathrm{n}=1667 ; \text { Idade: } 4-18 \\
\text { ơ }^{\top} 859 \text { e 우 } 808\end{array}$ & $\begin{array}{l}\text { Prev total na amostra: } 4 \% \\
\text { Prev em Sp: } 15.4 \%\end{array}$ \\
\hline \multirow[t]{2}{*}{$\begin{array}{l}\text { Calcaterra } \\
\text { et al (10) }\end{array}$} & $\begin{array}{l}\text { Prev. de SM em crianças e } \\
\text { adolescentes obesas }\end{array}$ & Itália & $\begin{array}{l}\text { NCEP ATP III e OMS } \\
\text { (adaptado) }\end{array}$ & $\begin{array}{c}\mathrm{n}=191 ; \text { ldade: } 11-15 \\
\text { ơ }^{7} 93 \text { e 우 } 98 \\
\text { Caucasianas }\end{array}$ & Prev total na amostra: $13.9 \%$ \\
\hline & & \multicolumn{3}{|c|}{ Score Z - Score Contínuo da Síndrome Metabólica } & \\
\hline $\begin{array}{l}\text { Dubose } \\
\text { et al (16) }\end{array}$ & $\begin{array}{c}\text { Influência do IMC e da AptF } \\
\text { no score da SM em crianças } \\
\text { com peso normal, em risco } \\
\text { de Sp e com Sp }\end{array}$ & EUA & $\begin{array}{l}\text { NCEP ATP III } \\
\text { (adaptado) }\end{array}$ & $\begin{array}{c}\text { n=375; Idade: } 7-9 \\
\text { ऽ } 182 \text { e 우 } 193 \\
27 \% \text { outros que } \\
\text { não - hispânicos brancos }\end{array}$ & $\begin{array}{c}\text { Obesidade e score SM } \\
r=0.7 p<0.05 \\
\text { AptF e score SM } \\
r=-0.46 p<0.05\end{array}$ \\
\hline $\begin{array}{l}\text { Olireira } \\
\text { et al ( } 40)\end{array}$ & $\begin{array}{l}\text { Avaliar a associação entre a } \\
\text { SM e as suas componentes } \\
\text { com a Proteína C-reactiva }\end{array}$ & Brasil & NCEP ATP III & $\begin{array}{c}\mathrm{n}=407 ; \text { Idade média } 11.3 \\
\text { б' } 273 \text { e 우 } 229 \\
72 \text { com SM; } 335 \text { sem SM }\end{array}$ & $\begin{array}{l}\text { OR } 7.9 ; 4.7 ; 2.3 ; p<0.05 \\
\text { Respectivamente para } \\
\text { Excesso de peso, hipertensão } \\
\text { arterial e hipertriglideridemia. }\end{array}$ \\
\hline \multirow[t]{2}{*}{$\begin{array}{l}\text { Hirschler } \\
\text { et al (26) }\end{array}$} & $\begin{array}{c}\text { Determinar a associação } \\
\text { entre } P C \text {, e o score e todos os } \\
\text { componentes da SM }\end{array}$ & Argentina & NCEP ATP III & $\begin{array}{c}\mathrm{n}=84 \text {; Idade média: } 6-13 \\
\sigma^{\nearrow} 40 \text { e 우 } 44 \\
68 \text { obesos ou com Sp }\end{array}$ & $\begin{array}{c}\mathrm{PC}(\mathrm{r}=0.73) ; \mathrm{IMC}(\mathrm{r}=0,96) ; \\
\text { Estádio de maturação de } \\
\text { Tanner }(\mathrm{r}=0.56) ; \text { idade } \\
\text { (r=0.56); PA Sist }(\mathrm{r}=0,64) ; \mathrm{PA} \\
\text { Diast }(\mathrm{r}=0.61) ; \mathrm{C}-\mathrm{HDL}(0.45)\end{array}$ \\
\hline & & & Análise Factorial (AF) & & \\
\hline $\begin{array}{l}\text { Edwards } \\
\text { et al (17) }\end{array}$ & $\begin{array}{l}\text { Traduzir todos os factores de } \\
\text { risco da SM a um número } \\
\text { reduzido de factores que tra- } \\
\text { duzam a mesma informação }\end{array}$ & EUA & OMS & $\begin{array}{c}\mathrm{n}=704 ; \text { Idade: } 49.6 \pm 12.6 \\
90 \% \text { caucasianas } \\
\text { Gémeas do Kaiser } \\
\text { Permanent Women Twin } \\
\text { Study (Oakland) } 1990\end{array}$ & $\begin{array}{l}\text { Redução dos } 10 \text { factores } \\
\text { de risco a } 3 \text { factores que } \\
\text { explicam } 66 \% \text { da variância }\end{array}$ \\
\hline $\begin{array}{l}\text { Young } \\
\text { et al (61) }\end{array}$ & $\begin{array}{c}\text { Comparar os padrões de } \\
\text { associação dos factores de } \\
\text { risco da SM e verificar como } \\
\text { a expressão do seu fenótipo } \\
\text { varia consoante diferentes } \\
\text { etnias }\end{array}$ & Canadá & $\begin{array}{c}\mathrm{n} / \mathrm{a} \\
\text { Definição da Diabetes: } \\
\text { ADA (1997) }\end{array}$ & $\begin{array}{c}\mathrm{n}=3930 ; \text { Idade: } 38-48 \\
\text { Índios, inuítes e } \\
\text { não aborígenas } \\
\text { - canadianos }\end{array}$ & $\begin{array}{l}\text { Os factores "obesidade", "PA" } \\
\text { e "Lípidos/Glucose" explica- } \\
\text { ram 64,3\% da variância. } \\
\text { Os Índios apresentaram os } \\
\text { scores mais elevados dos } 3 \\
\text { factores. }\end{array}$ \\
\hline $\begin{array}{l}\text { Austin } \\
\text { et al (?) }\end{array}$ & $\begin{array}{l}\text { Caracterizar a SM numa } \\
\text { população específica, } \\
\text { estimar a heritabilidade } \\
\text { dos factores extraídos }\end{array}$ & EUA & $\mathrm{n} / \mathrm{a}$ & $\begin{array}{c}\mathrm{n}=432 \text {; Idade: } 54.2 \pm 15.8 \\
\text { ơ } 190 \text { e 우 } 242 \\
\text { Sem Diabetes } \\
\text { Nipo-Americanos }\end{array}$ & $\begin{array}{l}F 1: h^{2}=0.52, p<0.001 \\
F 2: h^{2}=0.27, p=0.016 \\
F 3: h^{2} 0.25, p=0.026\end{array}$ \\
\hline
\end{tabular}

Abreviaturas: SM - Síndrome metabólica; EUA - Estados Unidos da América; Prev - Prevalência; PC - Perímetro de Cintura; Sp - Sobrepeso; IMC - índice de Massa Corporal; AptF - Aptidão Física; PA - Pressão Arterial; Sist - Sistólica; Diast - Diastólica; C - HDL - Colesterol com Lipoproteina de Alta Densidade; ADA - American Diabetes Association; F1: factor - Lípidos; F2: factor - gordura corporal, glucose e proteína C-reactiva; F3: factor-pressão sanguínea; h2 heritabilidade 
Tabela 5. Sumário de estudos recentes, em crianças e adultos, sobre a relação entre a actividade física e a SM assim como a influência de possíveis factores confundidores.

\begin{tabular}{|c|c|c|c|c|c|}
\hline $\begin{array}{l}\text { Autor } \\
\text { (data) }\end{array}$ & $\begin{array}{l}\text { Objectivos } \\
\text { do estudo }\end{array}$ & País & Amostra & $\begin{array}{l}\text { Instrumentos } \\
\text { de avaliação }\end{array}$ & $\begin{array}{l}\text { Principais } \\
\text { resultados }\end{array}$ \\
\hline $\begin{array}{l}\text { Brage } \\
\text { et al (9) }\end{array}$ & $\begin{array}{l}\text { Avaliar a relação entre a SM e } \\
\text { os níveis de AF e verificar até } \\
\text { que ponto a alteração desses } \\
\text { níveis faz variar esta relação. }\end{array}$ & Alemanha & $\begin{array}{l}n=589 \text { crianças } \\
\sigma^{\nearrow}=279 ; \text { 우 }=310 \\
\text { ldade }=9,6 \pm 0,44\end{array}$ & $\begin{array}{l}\text { AF: } \\
\text { Acelerómetro; } \\
\text { ACR: } \\
\text { Cicloergómetro }\end{array}$ & $\begin{array}{c}\text { A SM está inversamente relaciona- } \\
\text { da com a AF }(p<0,008) \text {. A relação } \\
\text { ficou atenuada quando se ajustou } \\
\text { à } A C R(p<0,127) .\end{array}$ \\
\hline $\begin{array}{l}\text { DuBose } \\
\text { et al (16) }\end{array}$ & $\begin{array}{c}\text { Avaliar a influência da ACR e } \\
\text { do IMC de crianças no score } \\
\text { de SM }\end{array}$ & EUA & $\begin{array}{l}n=375 \text { crianças } \\
\sigma^{\nearrow}=182 ; \text { 우 }=193 \\
\text { ldade }=7,7 \pm 0,7\end{array}$ & $\begin{array}{c}\text { ACR: } \\
\text { Cicoergómetro }\end{array}$ & $\begin{array}{c}\text { IMC relacionado directamente com } \\
\text { o score da } S M(r=0,70 ; p<0,001) \\
\text { A ACR modifica o impacto que } \\
\text { negativo que o IMC tem no score } \\
\text { da SM. }(p<0,05)\end{array}$ \\
\hline $\begin{array}{l}\text { Ekelund } \\
\text { et al (18) }\end{array}$ & $\begin{array}{l}\text { Avaliar a associação entre } \\
\text { diferentes níveis de AF e os } \\
\text { factores de risco da SM em } \\
\text { indivíduos com sobre-peso e } \\
\text { com história familiar de DM2 }\end{array}$ & $\begin{array}{l}\text { Reino } \\
\text { Unido }\end{array}$ & $\begin{array}{c}n=258 \text { adultos } \\
\sigma^{\nearrow}=103 ; \text { 우 }=155 \\
\text { Idade: } 30 \text { aos } 50 \text { anos } \\
\text { Com Sobre-peso } \\
\text { Com história familiar de DM2 }\end{array}$ & $\begin{array}{l}\text { AF: } \\
\text { Acelerómetro, } \\
\text { ACR: } \\
\text { Tapete rolante }\end{array}$ & $\begin{array}{l}\text { A AF está inversamente associada } \\
\text { ao score z da SM (após ajustar à } \\
\text { idade, sexo e obesidade). } \\
\text { Sedentários: } \mathrm{z}=0,07 \\
\text { AF ligeira: } \mathrm{z}=-0,03 \\
\text { AF moderada/alta: } \mathrm{z}=-0,09 \\
\text { Não houve diferenças significati- } \\
\text { vas após ajuste à ACR }\end{array}$ \\
\hline $\begin{array}{l}\text { Ruiz } \\
\text { et al (49) }\end{array}$ & $\begin{array}{c}\text { Avaliar a associação entre a } \\
\text { ACR e a SM e verificar se exis- } \\
\text { te um nível determinado de } \\
\text { ACR directamente relaciona- } \\
\text { do com um baixo score } z \text { da } \\
\text { SM }\end{array}$ & $\begin{array}{l}\text { Suécia e } \\
\text { Estónia }\end{array}$ & $\begin{array}{c}n=873 \text { crianças } \\
\sigma^{\nearrow}=429 ; \text { 우 }=444 \\
\text { Idade: } 9-10 \text { anos } \\
\text { Amostra proveniente do EYHS }\end{array}$ & $\begin{array}{c}\text { ACR: } \\
\text { Cicloergometro }\end{array}$ & $\begin{array}{c}\text { Associação inversa entra ACR e a } \\
\text { SM }(p<0,001) \\
0 \text { nível de ACR das crianças para } \\
\text { um score } z \text { baixo de } S M \text { foi de } \\
\text { 우 } 37.0 \mathrm{~mL} / \mathrm{kg} / \mathrm{min} \\
\text { ○ } 42,1 \mathrm{~mL} / \mathrm{kg} / \mathrm{min}\end{array}$ \\
\hline $\begin{array}{l}\text { Rizzo } \\
\text { et al (48) }\end{array}$ & $\begin{array}{l}\text { Avaliar a influência entre } \\
\text { diferentes níveis de AF e ACR } \\
\text { na SM, tendo em conta a \% de } \\
\text { massa gorda, idade e sexo }\end{array}$ & Suécia & $\begin{array}{c}\mathrm{n}=273 \text { crianças } \\
\sigma^{\nearrow}=141 ; \text { 우 }=132 \\
\text { Idade: } 9,6 \pm 0,4 \\
n=256 \text { adolescentes } \\
\sigma^{\nearrow}=123 ; \text { 우 }=133 \\
\text { Idade: } 15,7 \pm 0,4 \\
\text { Amostra proveniente do EYHS }\end{array}$ & $\begin{array}{l}\text { AF: } \\
\text { Acelerómetro, } \\
\text { ACR: } \\
\text { Cicloergómetro }\end{array}$ & $\begin{array}{l}\text { Relação inversa entre AF e SM } \\
\text { AF Total associada à SM nas crian- } \\
\text { ças ( } \sigma^{7} \text { e 우 ] e nos adolescentes } \\
\text { (우) }(p<0,006) \text {; } \\
\text { AF Moderada e Intensiva associa- } \\
\text { da à SM em ambos os sexos e gru- } \\
\text { pos etários ( } p<0,036 \text { e } p<0,047 \text { ) } \\
\text { No grupo com baixo nível de AF a rela- } \\
\text { ção enfraqueceu quando se ajustou à } \\
\text { ACR e \% de gordura corporal }\end{array}$ \\
\hline $\begin{array}{l}\text { Healy } \\
\text { et al (25) }\end{array}$ & $\begin{array}{c}\text { Examinar a associação entre } \\
\text { a inactividade física e o score } \\
\text { da SM }\end{array}$ & Austrália & $\begin{array}{c}n=169 \text { adultos } \\
\sigma^{\nearrow}=67 ; \text { 우 }=102 \\
\text { Idade: } 30 \text { aos } 87 \text { anos } \\
\text { Sem DM2 }\end{array}$ & $\begin{array}{c}\text { AF: } \\
\text { Acelerómetro }\end{array}$ & $\begin{array}{c}\text { Verificou-se uma associação signi- } \\
\text { ficativa entre as variáveis da acti- } \\
\text { vidade física e o } P C+S M \text {. } \\
\text { SM: Sedentários: } z=0,23 \\
\text { AF ligeira: } z=-0,20 \\
\text { AF moderada/alta: } z=-0,17 \\
\text { PC: Sedentários: } r=0,22 \\
\text { AF ligeira: } r=-0,20 \\
\text { AF moderada/alta: } r=-0,16 \\
\end{array}$ \\
\hline $\begin{array}{l}\text { Yang } \\
\text { et al (59) }\end{array}$ & $\begin{array}{c}\text { Avaliar a relação entre a AF e } \\
\text { as suas alterações ao longo } \\
\text { de } 9 \text { anos (dados recolhidos } \\
\text { entre } 1992 \text { e } 2001 \text { ) com a } \\
\text { prevalência da SM }\end{array}$ & $\overline{\text { Finlândia }}$ & $\begin{array}{c}\mathrm{N}=2060 \text { jovens adultos } \\
\sigma^{\nearrow}=961 ; \text { 우 }=1099 \\
\text { Em } 1992 \\
\text { Idade: } 15 \text { aos } 30 \\
\text { Em } 2001: \\
\text { Idade: } 24 \text { aos } 39 \\
\text { Sem DM2 nem DCV }\end{array}$ & AF: Questionário & $\begin{array}{l}\text { Baseline: Relação Linear em ठ } \\
\text { entre a SM e AF }(p<0,01) \\
\text { F-up: As alterações nos níveis de } \\
\text { AF ao longo dos } 9 \text { anos tiveram } \\
\text { impactos no score z da SM em } \\
\text { ambos os sexos. } \\
\text { A relação entre AF e SM é inversa e } \\
\text { significativa mesmo após ajuste a } \\
\text { efeitos confundidores (idade, hábitos } \\
\text { tabágicos e nível de educação) }\end{array}$ \\
\hline
\end{tabular}


autores (ou entidades) selecciona um deles (como específico) e mais dois ou três para ser realizado o diagnóstico da síndrome metabólica. Será de pensar que todas as combinações possíveis (com 5 componentes temos 32 combinações, com 6 temos 64 combinações) entre esses mesmos componentes têm o mesmo peso clínico(31). Ou seja, será que existem combinações com mais significado que outras? Será que cada um dos factores revela um valor preditivo diferente se estiver igualmente agrupado com diferentes factores? Será que esses mesmos factores agrupados, aumentam o risco de doença cardiovascular em comparação com a soma do seu risco isoladamente? Quais os procedimentos estatísticos a utilizar para tentar responder a estas questões?

A estas dúvidas, somam-se outras às quais urge a necessidade de resposta permitindo assim a adaptação de conhecimentos à prática clínica em benefício do doente.

\section{RELAÇÃO ENTRE A (IN)ACTIVIDADE FÍSICA E CONTINUUM DE RISCO METABÓLICO}

É do conhecimento geral que a população mundial é cada vez mais sedentária e adopta estilos de vida cada vez menos saudáveis. Esta é uma preocupação que atinge principalmente os países desenvolvidos que continuamente se esforçam por divulgar e alertar as populações para o benefício do exercício físico, de uma alimentação equilibrada e para a adopção de estilos de vida saudáveis. Infelizmente os resultados dos inúmeros estudos nem sempre têm o impacto desejado uma vez que existe ainda um conjunto muito alargado de pessoas em vários países, que, além de não adoptarem estilos de vida saudáveis, têm valores de actividade física abaixo do recomendado $(9,39,50,55,57,58)$.

A relação entre os níveis de actividade física e a prevalência da SM em adultos tem sido alvo de pesquisas intensivas. A maioria dos estudos encontrados identifica uma associação inversa entre os níveis de actividade física e a prevalência ou incidência desta enfermidade ${ }^{(5}$, 9, 18, 25, 41, 48, 49, 50, 54, 59). Esta associação inversa sustenta promoção da prática de exercício físico regular (com nível moderado a intenso) como uma estratégia de eleição para prevenção e tratamento da $\mathrm{SM}^{(23)}$.

A afinidade entre a (in)actividade física e a síndrome metabólica é igualmente encontrada quando nos referimos a populações pediátricas (ver Tabela 5). Alguns estudos relatam o impacto que níveis diferenciados de actividade física têm no valor compósito da agregação dos factores de risco metabólico, sobretudo a sua importância como factor protector da doença $(16,41,50,59)$. Contudo, os resultados encontrados variam em função de ajustamentos estatísticos a factores aditivos ou de confundimento.

Características pessoais como idade, sexo, etnia e estilo de vida são elementos que poderão interferir nos resultados das análises estatísticas e, por isso mesmo, não devem ser esquecidos. Além disso, o IMC, a aptidão cardio-respiratória e o exercício físico deverão ser igualmente descritos em detalhe, de forma isolada ou conjuntamente, para que os resultados não sejam "contaminados" pela sua covariação com os níveis de actividade física. Alguns dos estudos encontrados, resumidos na Tabela 5, fazem essa análise e em alguns casos os resultados são ligeiramente diferentes quando se efectuam ajustamentos para confundidores. Se umas vezes a relação AF e SM é enriquecida quando se adicionam covariáveis como aptidão cardio-respiratória, também poderá perder significado quando se ajustam para outras como idade ou o IMC.

Tal como demonstra a tabela anterior, a relação inversa entre a AF e a SM foi encontrada em diversos estudos em vários países. Os mesmos achados são encontrados em Portugal. Recentemente foi realizado um estudo na Região Autónoma dos Açores por Campos ${ }^{(11)}$ com o objectivo de avaliar a importância da actividade física regular na manifestação das componentes da síndrome metabólica. Os dados obtidos foram coerentes com a maioria das pesquisas realizadas a nível mundial, revelando que, tanto em adultos como em crianças, existe uma associação significativa e negativa entre a actividade física e as componentes da SM. As crianças e jovens menos activos possuem maior prevalência nos indicadores desta enfermidade e revelam uma propensão superior para a manifestação de risco metabólico. Assim, a agregação de factores de risco metabólico está associado aos níveis distintos de actividade física. Daqui que os níveis moderados a elevados de actividade física tenham um efeito protector da doença. Além disso, é de reforçar que, o exercício físico é actualmente considerado um dos meios de eleição 
para a prevenção de evento cardiovascular. O Colégio Americano de Medicina Desportiva considera o exercício físico como um "medicamento", ao ponto do seu slogan actual ser - exercise is medicine. Salienta-se a necessidade de assumir o exercício físico como um fármaco (profilático ou terapêutico), que deve ser prescrito na dosagem precisa com a frequência adequada e própria para cada indivíduo.

\section{CONCLUSÃO}

As pesquisas referenciadas mostram a relevância que esta síndrome apresenta no que diz respeito aos impactos para o doente, família e para a própria economia (familiar e do país). A definição da síndrome metabólica passou por várias etapas ao longo do tempo, até ao formato actual. É consensual o facto de resultar de um agrupamento (clusters) de vários factores de risco cardiovascular, mostrando em termos populacionais um aumento quase exponencial do risco cardiovascular e prevalência de patologia. A aplicabilidade clínica dos critérios da SM nem sempre é clara, porque na maioria dos casos a própria definição desta síndrome incita à sua redução a um diagnóstico binário: "ter" ou "não ter". É através da dicotomização das variáveis e da conjugação combinatória de factores de risco com valores de corte rígidos, que é feito o diagnóstico. Este procedimento básico pode limitar a análise da magnitude risco em função das múltiplas configurações de diferentes factores do risco, em função do peso que cada um representa isoladamente ou agrupado a outros, em função dos valores quantitativos de cada um dos factores. Torna-se pois desejável o recurso a vários métodos de análise estatística para melhor perceber a magnitude e a relevância distinta da influência dos diversos factores genéticos e ambientais nos valores desta síndrome.

É hoje claro o papel claro que a actividade física desempenha na manutenção da saúde. O efeito protector que níveis moderados a elevados de actividade física regular exercem nos factores de risco metabólico é óbvio e demonstrado por vários autores. Da mesma forma, a inactividade física e o sedentarismo revelam uma forte associação com a síndrome metabólica assim como potencia o aumento do risco cardiovascular. Desta forma torna-se mais uma vez evidente a importância fulcral que o exercício físico tem na pre- venção e como adjuvante no tratamento da síndrome metabólica.

\section{AGRADECIMENTO}

Ao editor da RPCD pelas críticas e sugestões que permitiram melhorar substancialmente o trabalho. Este texto decorre de um projecto de pesquisa financiado pela FCT sob o número PTDC/DES/67569/2006 designado actividade física, aptidão física associada à saúde, somatótipo e síndrome metabólica. Um estudo longitudinal em famílias nucleares.

\section{CORRESPONDÊNCIA}

\section{José António Ribeiro Maia}

Laboratório de Cineantropometria e Estatística Aplicada

Faculdade de Desporto da Universidade do Porto

Rua Dr. Plácido Costa, 91

4200-450 Porto - Portugal

E-mail: jmaia@fade.up.pt 


\section{REFERÊNCIAS}

1. Administração Central do Sistema de Saúde, I.P. (2006). Sistema Classificação de Doentes em Grupos de Diagnósticos Homogéneos (GDH). Consult 18 Ago 2008, disponível em http:/ /www.acss.min-

saude.pt/Portals/0/DownloadsPublicacoes/SNS/Info_Activi d/Rel_Nacional_06.pdf

2. Albert F, Zimmet P (1998). Definition, diagnosis and classification of diabetes mellitus and its complications - Part 1: Diagnosis and classification of diabetes mellitus, provisional report of WHO consultation. Diabet Med, 15: 539553.

3. Alberti K, Zimmet P, Shaw J (2006). Metabolic Syndrome e new world-wide definition. A consensus statement from the International Diabetes Federation. Diabetic Medicine, 23: 469-480.

4. American College of Endocrinology (2003). Position statement on the insulin resistance syndrome. Endocr Pract, 9: 236-252.

5. Andersen L, Harro M, Sardinha L, Froberg K, Ekelund U, Brage S, et al (2006). Physical Activity and clustered cardiovascular risk in children: a cross sectional study. Lancet, 368: 299-304.

6. Arya R, Blangero J, Wolliams K, Almasy L, Dyer T et al. (2002). Factors of insulin resistance syndrome-related phenotypes are linked to genetic locations on chromosomes 6 and 7 in nondiabetic Mexican-Americans. Diabetes, 51: 841847.

7. Austin M, Edwards K, McNeely M, Chandler W, Leonetti D, Talmud P et al (2004). Brief genetics report: heritability of multivariate factors of the metabolic syndrome in nondiabetic Japanese American. Diabetes, 53: 1166-1169.

8. Balkau B, Charles M (1999). Comment on the provisional report from the WHO consultation - European Group for The study of Insulin Resistance (EGIR). Diabet Med, 16(5): 442-43.

9. Brage S, Wedderkopp N, Ekelund U, Franks P, Wareham N, Anderson L et al (2004). Features of the metabolic syndrome are associated with objectively measured in physical activity and fitness in Danish children. Diabetes Care, 27: 2141-2148.

10. Calcaterra V, Klersy C, Muratori T, Telli S, Caramagna C, Scaglia F et al (2008). Prevalence of metabolic syndrome (MS) in children and adolescents with varying degrees of obesity. Clinical Endocrinology, 68(6): 868-72.

11. Campos MAOA (2007). Importância da Actividade Física e o risco de Síndrome Metabólica. Dados de Crianças, Jovens, Adultos e Famílias da Região Autónoma dos Açores. Porto: P. Fonte. Dissertação de Mestrado apresentada à Faculdade de Desporto da Universidade do Porto.

12. Carr D, Utzschneider K, Hull R, Kodoma K, Retzlaff B, Brunzell J et al. (2004). Intra-abdominal fat is a major determinant of the National Cholesterol Education Program Adult Treatment Panel III Criteria for the metabolic syndrome. Diabetes, 53(8): 2087-2094.

13. Cook S, Weitzman M, Auringer P, Nguyen M, Dietz W (2003). Prevalence of a Metabolic Syndrome - Phenotype in Adolescents. Arch Pediatric Adolesc Med, 157: 821-827.

14. Cruz M, Weigensberg M, Huang T, Ball G, Shaibi C, Goran M (2004). The Metabolic Syndrome in Overweight Hispanic Youth and the role of insulin Sensitivity. The Journal of Clinical Endocrinology \& Metabolism, 89(1):108-113.
15. Dekker J, Girman C, Rhodes T, Nijpels G, Stehouvwer C, Bouter L et al. (2005). Metabolic syndrome and 10-year cardiovascular disease risk in the Hoorn Study. Circulation, 112: 666-673.

16. Dubose K, Eisenmann J, Donnelly J (2007). Aerobic fitness attenuates the metabolic syndrome score in normal-weight, at-risk-for-overweight, and overweight children. Pediatrics, 120(5): 1262 - 1268.

17. Edwards K, Austin M, Newman B, Mayar E, Krauss R, Selby J (2006). Multivariate analysis of insulin resistance syndrome in women. Arteriosclerosis, thrombosis, and vascular biology, 14(12): 1940-1945.

18. Ekelund U, Wareham N, Griffin S (2007). Physical Activity and Metabolic Risk in Individuals with a Family History of Type 2 Diabetes. Diabetes Care, 30(2): 337-342.

19. Expert Panel on Detection, Evaluation, and Treatment of high blood cholesterol in adults (Adult Treatment Panel III). (2001). Executive Summary of the Third Report of the National Cholesterol Education Program (NCEP). JAMA, 285: $2486-2497$

20. Ferranti SD, Gauvreau K, Ludwig DS, Neufeld EJ, Jane W (2004). Prevalence of the Metabolic Syndrome in American Adolescents: Findings From the Third National Health and Nutrition Examination Survey. Circulation, 110: 2494-2497

21. Franks P, Olsson T (2007). Metabolic Syndrome and Early Death: Getting to the Heart of the Problem. Hypertension, 49: $10-12$

22. Gonzalez R, Piaza E, Moreno J (1997). Dicionário de Ciências da Saúde. Lisboa (Portugal): Ed McGraw-Hill

23. Grundy S, Cleeman J, Daniels S, Donato A, Eckel R, Krauss $\mathrm{R}$ et al (2005). Diagnosis and management of the metabolic syndrome. An American Heart Association/National Heart, Lung and blood institute. Scientific Statement. Circulation, 112: 2735-2752

24. Hayakawa T, Takamura T, Abe T, Kaneko S (2007). Association of the C825T polymorphism of the G-protein 3 sub-unit gene with hypertension, obesity, hiperlipidemia, insulin resistance, diabetes, diabetic complications and diabetic therapies among Japanese. Metabolism Clinical and Experimental, 56: 44-48

25. Healy G, Wijndaele K, Zimmet P, Dunstan D, Shaw J, Salmon J, Owen N (2008). Objectively measured sedentary time, physical activity, and metabolic risk. Diabetes Care, 31(2): 369-371

26. Hirschler V, Aranda C, Calcagno M, Maccalini G, Jadzinsky M (2005). Can waist circumference identify children with the metabolic syndrome? Arch Pediatr Adolesc Med, 159: 740-744

27. Huang T, Ball G, Franks P (2007). Metabolic Syndrome in youth: Current issues and challenges. Appl physiol nutre Metab, 32: 13-22

28. INE (2006). Indicadores e Metas do PNS. Consult. 20 Nov 2008, disponível em http://www.acs.minsaude.pt/pns/pt/doencas-cardiovasculares/

29. Jebb S, Lang R, Penrose A (2003). Improving communication to tackle obesity in the UK. Proc Nutr Soc 62 (3): 57781.

30. Jeppesen J, Hansen T, Resmussen S, Ibsen H, Pedersen C, Madsbad S (2007). Insulin resistance, the metabolic syndrome, and the risk of incident cardiovascular disease - a population-based study. J Am Coll Cardiol 49(21): 2112-9.

31. Kahn R, Ferrannini E, Buse J, Stem M (2005). The metabo- 
lic syndrome: time for a critical appraisal. Diabetes care, 28: 2289-304

32. Klein B, Klein R, Bek K (2002). Components of the metabolic syndrome and risk of cardiovascular disease and diabetes in Beaver Dam. Diabetes Care, 25: 1790-94

33. Leal J, Luengo-Fernandez R, Gray A, Petersen S, Rayner M (2006). Economic burden of cardiovascular diseases in the enlarged European Union. European Heart Journal, 27: 16101619

34. Lin H, Boden Albala B, Juo S, Park N, Rundek T, Sacco R (2005). Heritabilities of the metabolic syndrome and its components in the northern Manhattan Family Study. Diabetologia, 48: 2006-2012

35. Magder L, Fix A (2003). Optimal Choice of a cut point for a quantitative diagnostic test performed for research purposes. Journal of Clinical Epidemiology, 56: 956-962

36. Meigs J, Wilson P, Nathan D, D’Agostino R, Williams K, Haffner S (2003). Prevalence and characterising metabolic syndrome in the San Antonio Heart and Framingham Offspring Studies. Diabetes 62: 2160-2167

37. Meirhaeghe A, Cottel D, Amouyel P, Dallongeville J (2005). Association between Peroxisome Proliferator-Activated Receptor haplotypes and the metabolic syndrome in French Men and Women. Diabetes, 54: 3043-3047

38. Millen B, Pencina M, Kimikoti R, Zhu L, Meigs J, Ordovas J et al (2006). Nutritional risk and metabolic syndrome in women: opportunities for preventive intervention from the Framingham nutrition Study. American Journal of clinical nutrition, 84(2): 434-441

39. Misra K, Endemann S, Ayer M (2005). Leisure time physical activity and metabolic syndrome in Asian Indian immigrants residing in Northern California. Ethnicity \& Disease, 15(4): 627-634

40. Oliveira AC, Oliveira AM, Adan L, Oliveira N, Silva A, Ladeia A (2008). C-reactive protein and metabolic syndrome in youth: A strong relationship? Obesity (Silver Spring) 16(5): 1094-1098

41. Pan Y, Pratt CA (2008). Metabolic Syndrome and Its Association with Diet and Physical Activity in US Adolescents. J Am Diet Assoc, 108:276-286

42. Picon P, Zanatta C, Gerchman F, Zelmanovitz T, Gross J, Canani L (2006). Análise dos critérios de definição da Síndrome metabólica em pacientes com diabetes melito tipo 2. Arq Brás endocrinol Metab, 5: 264-270

43. Ravaglia G, Forti P, Maioli F, Bastagli L, Chiappelli M, Montesi F et al (2006). Metabolic Syndrome: prevalence and prediction of mortality in elderly individuals. Diabetes Care, 29: 2471-2476

44. Reaven GM (1988). Banting lecture 1988: role of insulin resistance in human disease. Diabetes, 37: 1596-607

45. Reaven GM (2005). The Metabolic Syndrome: Requiescat in Pace. Clinical Chemistry, 51(6): 931-938

46. Rennie K, McCarthy N, Yazdgerdi S, Marmot M, Brunner E (2003). Genetic Versus environmental etiology of metabolic syndrome with both vigorous and moderate physical activity. International Journal of Epidemiology, 32: 600-606

47. Rezende JM (2002). Linguagem médica. (2. ${ }^{a}$ edição). Goiás, Brasil: Editora Gegraf.
48. Rizzo N, Ruiz J, Hurting-Wwnnlof A, Ortega F, Sjostrom M (2007). Relationship of physical activity, fitness, and fatness with clustered metabolic risk in children and adolescents: the European Youth Heart Study. The Journal of Pediatrics, 150(4): 388-394

49. Ruiz J, Ortega F, Rizzo N, Villa I, Hurtig-Vennlöf A, Oja L, SJöström M (2007). Hight cardiovascular fitness is associated with low metabolic risk score in children: The European Youth Heart Study. Pediatr Res 61: 350-355

50. Steele RM, Brage S, Corder K, Wareham NJ, Ekelund U (2008). Physical activity, cardiorespiratory fitness, and the metabolic syndrome in youth. Appl Physiol, 105: 342-351

51. Terán-Garcia M, Bouchard C (2007). Genetics of the metabolic syndrome. Appl Physiol Nutr Metab, 32: 89-113

52. Wannamethee S, Shaper A, Wuincup H (2006). Modifiable lifestyle factors and the metabolic syndrome in older men: effects of lifestyle changes. The American Geriatrics Society, 54(12): 1909-1914

53. Weiss R, Dziura J, Burgert T, Tamborlane W, Taksali S, Yeckel $C$ et al (2004). Obesity and the metabolic syndrome in children and adolescents. N Engl J Med, 350: 2362-2374

54. Wijndaele K, Beunen G, Matton L, Duquet W, Lefevre J, Philippaerts R (2006). A continuous Metabolic Syndrome Risk Score: utility for Epidemiological analyses. Diabetes Care, 29(10): 2329

55. Wijndaele K, Duvigneaud L, Matton L, Duquet W, Thomis M, Beunen G, Philippaerts R (2007). Muscular Strenght, aerobic fitness and Metabolic Syndrome risk in Flemish Adults. Medicine \& Science in Sports \& Exercise, 39: 233-240

56. WHO (1999). Definition, Diagnosis and Classification of Diabetes Mellitus and its complications. Report of a WHO consultation. Genève.

57. WHO (2004). The atlas of heart disease and stroke. Consult 23 Out 2008 Disponível em http://www.who.int/cardiovascular diseases/resources/atlas/en/index.html

58. WH̄O (2007). Cardiovascular Diseases. Consult 23 Out 2008, disponível em http://www.who.int/mediacentre/factsheets/ fs317/en/index.html

59. Yang X, Telama R, Hirvensalo M, Mattsson N, Viikari J, Raitakari O (2008). The Longitudinal Effects of Physical Activity History on the Metabolic Syndrome. Medicine $\mathcal{E}$ Science in Sports \& Medicine. 40(8): 1424-1431

60. Yoshinaga M, Tanaka S, Shimago A, Sameshima K, Nishi J, Nomura Y, Kawano Y, Hashiguchi J, Ichiki T, Shimizu S (2005). Metabolic syndrome in overweight and obese Japanese children. Obes Res 13(7): 1135-40

61. Young T, Chateau D, Zhang M(2002). Factor analysis of ethnic variation in the multiple metabolic (insulin resistance) syndrome in three Canadian populations. Am J Hum Biol 14: 649-58

62. Zimmet P, Magliano D, Matsuzawa Y, Alberti G, Shaw J (2005). The metabolic syndrome: A global public health problem and a new definition. Journal of atherosclerosis and thrombosis, 12(6): 295-300

63. Zimmet P, Alberti G, Kaufman F, Tajima N, Silink M, Arslanian S. (2007). The Metabolic Syndrome in Children and Adolescents. The Lancet, 369: 2059-61 